\title{
Apoptosis in parasites and parasite-induced apoptosis in the host immune system: a new approach to parasitic diseases
}

M.A. Barcinski1 and G.A. DosReis ${ }^{2}$

\author{
${ }^{1}$ Departamento de Parasitologia, Instituto de Ciências Biomédicas, \\ Universidade de São Paulo, São Paulo, SP, Brasil \\ ${ }^{2}$ Programa de Imunobiologia, Instituto de Biofísica Carlos Chagas Filho, \\ Universidade Federal do Rio de Janeiro, Rio de Janeiro, RJ, Brasil
}

\author{
Correspondence \\ M.A. Barcinski \\ Departamento de Parasitologia \\ ICB, USP \\ Av. Prof. Lineu Prestes, 1374 \\ 05508-900 São Paulo, SP \\ Brasil \\ Fax: + 55-11-818-7417 \\ E-mail: barcinsk@biomed.icb2.usp.br \\ Research supported by grants from \\ PADCT (No. 62.0314/94.0), CNPq, \\ FINEP, and PRONEX (No. 026/1995), \\ Ministry of Science and Technology, \\ Brazil, to G.A. DosReis, and by \\ grants from FAPESP and PRO NEX \\ (No. 41.96.0931.00) to \\ M.A. Barcinski.
}

Received February 16, 1998 Accepted February 4, 1999

\begin{abstract}
Apoptosis, a form of programmed cell death (PCD), has been described as essential for normal organogenesis and tissue development, as well as for the proper function of cell-renewal systems in adult organisms. Apoptosis is also pivotal in the pathogenesis of several different diseases. In this paper we discuss, from two different points of view, the role of apoptosis in parasitic diseases. The description of apoptotic death in three different species of heteroxenic trypanosomatids is reviewed, and considerations on the phylogenesis of apoptosis and on the eventual role of PCD on their mechanism of pathogenesis are made. From a different perspective, an increasing body of evidence is making clear that regulation of host cell apoptosis is an important factor on the definition of a host-pathogen interaction. As an example, the molecular mechanisms by which Trypanosoma cruzi is able to induce apoptosis in immunocompetent cells, in a murine model of Chagas' disease, and the consequences of this phenomenon on the outcome of the experimental disease are discussed.
\end{abstract}

\section{Introduction}

Apoptosis, a form of programmed cell death (PCD), a gene-regulated process of active cell death, plays a central role in normal tissue development and organogenesis (1) as well as in the pathogenesis of different diseases (2). PCD is responsible for the physiological non-inflammatory elimination of unnecessary or potentially harmful cells during organogenesis, and for the proper function of continuous cell-renewal systems in adult organisms. It has been clearly shown that, in higher vertebrates, PCD is pivotal for the normal development and function of the immune system. Both self-reactive maturing immunocompetent cells during the selective process and hyperreactive mature cells are eliminated by apoptosis. The Fas system, which shares a signal cascade with tumor necrosis factor (TNF) in one apoptotic pathway, can be useful for down-regulating immunoreactivity and for removing cancerous as well as virally infected cells (3). The therapeutic potential resulting from the controlled manipulation of the apoptotic process 
has raised great expectations in the biomedical community. Here, we discuss the recent findings of apoptosis among pathogenic protozoan parasites, and of apoptosis induced by parasites in the cells of the host immune system. The implication is that pharmacological and immunological manipulation of the apoptotic process could lead to a new therapeutic approach to chronic parasitic diseases.

\section{Apoptosis in unicellular pathogenic protozoan parasites}

Since cell death is the final outcome of apoptosis it is almost intuitive that this process serves only multicellular organisms. Indeed, if one considers a unicellular organism as a self-sufficient non-gregarious individual, there is no a priori reason to believe that such an organism can benefit from a suicidal program. A corollary of this way of thinking is that programmed cell death has evolved after the onset of multicellularity. Based on several lines of evidence from the literature showing that unicellular organisms can organize themselves as cell populations and establish patterns of intercellular communication $(4,5)$ and some of them can respond to growth factors and cytokines that are otherwise regulators of PCD (6), our group began to search for ultrastructural and biochemical evidence of apoptosis in promastigotes of Leishmania (L) amazonensis (7). In about $20 \%$ of the stationary phase promastigotes submitted to heat shock in the presence of calcium, we observed morphological alterations closely similar to apoptosis. These included chromatin clumping into nuclear lobes and nuclear fragmentation resembling pre-apoptotic bodies while the cytoplasmic organelles retained their normal morphology. An oligonucleosomal laddering pattern was observed in DNA extracted from these same parasites. No similar changes were found in parasites submitted to heat shock in the absence of calcium. At the same time, similar findings for two other pathogenic trypanosomatids were reported in the literature. Ameisen and collaborators (8) reported the developmental and induced apoptotic death of epimastigotes of Trypanosoma cruzi. Welburn and colleagues $(9,10)$ showed that the procyclic insect form of Trypanosoma brucei rhodesiense displays differential mRNA expression and undergoes apoptosis when treated in vitro with concanavalin A. The description of apoptosis in three examples of unicellular heteroxenic pathogenic kinetoplastids which alternate between vertebrate and invertebrate hosts as part of their life cycle raises some fundamental questions concerning the cellular biology and the evolutionary origin of PCD, its role in the emergence and maintenance of parasitism, the structure and function of the genes involved in the process, and the constraints of the multicellular organization for the proper operation of a cell death program.

In spite of the wide range of inducers and of the cell types in which PCD occurs, its basic morphological and biochemical features are always closely similar, strongly suggesting that a common effector mechanism is activated during the process. The evidence that mitochondrial dysfunction constitutes an early event of apoptosis has led Kroemer $(11,12)$ to postulate that mitochondria are the central organelles involved in the occurrence of PCD. Today we know that one of the elements involved in caspase activation is cytochrome c released by mitochondria (13). Promastigotes of Leishmania spp are protected from heat-shock by granulocyte-macrophage colony stimulating factor (GM-CSF), a hematopoietic growth-factor that also protects mammalian cells from death by apoptosis (14). An increase in cytosolic free calcium during heat shock was described in this form of the parasite (7). In this same stressing condition, impairment of mitochondrial and endoplasmic reticulum calcium uptake was observed in permeabilized para- 
sites. Furthermore, the use of carbonyl cyanide p-trifluoromethylphenyl-hydrazone (FCCP), a proton ionophore which disrupts the mitochondrial membrane potential, strongly sensitizes the parasite to heat and oxidative stress. In this situation the promastigotes are extraordinarily responsive to the protective effect of calcium chelators and GM-CSF, suggesting the involvement of mitochondria in the apoptotic process in this parasite form, and in the protective effect of the hematopoietic cytokine (Moreira MEC, Balanco JMF, Gadelha FR, Vercesi AE and Barcinski MA, unpublished results).

Based on his assumption that mitochondrial dysfunction is the central trigger of the apoptotic process, Kroemer (12) postulates that apoptosis may have appeared in evolution together with the endosymbiotic process that originated the eukaryotes and not with the onset of multicellularity. This is why many molecules involved in the apoptotic cascade are phylogenetically old. According to this same investigator, in spite of the occurrence of apoptosis in unicellular organisms, PCD as a developmentally regulated program is a prerogative of multicellular organisms. Our postulate is that multicellularity indeed underlies the occurrence of PCD but not necessarily in a single multicellular organism, rather, it can occur in any situation where living cells have developed structured relationships and division of functions $(6,15)$. Such organization is compatible with several different features of the life cycle of heteroxenic trypanosomatids (1618 ), and with their clonal population structure (19). In trypanosomatid evolution, heteroxenic life styles have appeared independently on more than one occasion (20). Whether apoptosis is a requisite for such an evolutionary event is still an open question which can be approached by looking for the occurrence of apoptosis in monoxenic and in free-living kinetoplastids, and this is one of the current lines of investigation in our laboratory.
What purpose could parasite apoptosis serve? In the insect vector it may be necessary for the control of the size of the parasite population. A strict population size control was shown in the case of Trypanosoma brucei in tsetse flies $(17,21)$, where procyclics displaying morphological features of apoptotic death were found in the midgut of refractory flies. Once established, the size of the parasite population remains constant for the fly's lifetime. This equilibrium, whereby parasite multiplication is balanced by regulated death, is mutually advantageous since parasite and fly are in competition for proline as an energy source. In the mammalian host, apoptosis may be useful for the parasite population in situations where an inflammatory reaction induced by dying parasites is to be avoided. Immune responses to protozoan parasites can be either inflammatory (Th1-type) and usually host-protective, or humoral (Th2type) and usually parasite-protective, and there is evidence of mutual antagonism between these types of response (22). A provocative speculation is that antigens from an infective apoptotic parasite could be preferential inducers of a host $\mathrm{CD} 4^{+} \mathrm{Th}-2 \mathrm{~T}$-cell response. Interestingly, one of the genes upregulated during $T$. brucei apoptosis codes for the homologue of LACK (Leishmania homologue of receptors for activated $\mathrm{C}$ kinase) (10), which has been described as a preferential target of an early Th-2 anti-Leishmania major T-cell response (23). As discussed later, recent evidence has shown that phagocytosis of apoptotic cells is a powerful anti-inflammatory signal that deactivates macrophages. Thus, another possibility is that apoptotic parasites deactivate macrophages when ingested. From a cell population perspective, apoptosis of a parasite subpopulation, following early infection of the host, could be employed by the parasites to evade early immune responses.

Finally, recognition of PCD in parasites permits a more comprehensive view of mechanisms of anti-parasite drug action, and 
suggests new therapeutic approaches. Oligonucleosomal DNA fragmentation has been recently described in a chloroquine-sensitive but not in a chloroquine-resistant strain of Plasmodium falciparum (24), raising the exciting possibility that some antiparasitic drugs could act by inducing apoptosis in their targets and that the molecular basis of drug resistance could lie in the inability of the drug to trigger the apoptotic machinery in the resistant parasite. Not only parasites undergo PCD. The cells of the host immune system also undergo PCD as a result of parasitic infection. Different lines of evidence point to the importance of PCD in the host response to infection.

\section{Host cell apoptosis in parasitic infections and its pathogenetic significance}

Recently accumulated evidence has shown that regulation of host cell apoptosis is a critical determinant factor in host-pathogen interactions. Microbial pathogens manipulate host cell apoptosis in different ways, either to increase their life span within infected cells, or to spread infection when triggered by environmental stimuli. Certain pathogenic DNA viruses actively inhibit apoptosis of their host cells (25), while measles virus induces apoptosis in dendritic and $T$ cells of the immune system (26). An increasing number of identified pathogenic bacteria rely on host macrophage apoptosis as a virulence mechanism (27). In addition, many infectious agents generate vigorous innate and acquired immune responses which are in turn, subjected to apoptotic mechanisms of containment. A delicate balance is mounted by the host so as to cope with the infectious process and, at the same time, to avoid the immunopathology that results from unwanted activity of effector leukocytes. Considering that effector cell activity is ultimately inhibited by immunoregulatory circuits, it is possible that the balance achieved is in many cases responsible for the chronification of infectious states.

Chagas' disease is caused in humans by chronic infection with the protozoan parasite Trypanosoma cruzi. Recently, preliminary evidence was presented in a canine model of Chagas' disease myocarditis that apoptosis is abundant in the inflammatory infiltrates of the heart, affecting lymphocytes, infected and uninfected myocytes, and even parasites (28). This finding illustrates how important PCD can be in the immunopathology of Chagas' disease, and how it applies to parasites, lymphoid cells and myocytes, all in the same inflamed target tissue. The basic features of T. cruzi-induced lymphocyte PCD are being investigated in murine models of experimental Chagas' disease.

Infection of mice with $T$. cruzi leads to readily demonstrable ex vivo $\mathrm{CD}^{+}$and $\mathrm{CD} 8^{+}$ T-lymphocyte apoptosis in spleen (29). Lymphocyte apoptosis may derive from the powerful polyclonal lymphocyte activation triggered by the infection, i.e, it may result from the host's own regulatory needs (30). This proposal is consistent with the propriocide hypothesis of mature T-cell apoptosis, following engagement of the T-cell receptor (TCR) in actively cycling cells (31). A previous study showed that treatment of mice with hydroxyurea, which eliminates cycling cells, was able to ameliorate T-cell suppression in the acute infection (32). This finding indicates that suppressive mechanisms are indeed coupled to polyclonal lymphocyte activation. We have previously demonstrated that lymphocyte PCD in T. cruzi infection is responsible for in vitro T-cell unresponsiveness to stimuli that engage the TCR (33). One likely molecular link between lymphocyte cycling and immunosuppression is the Fas (CD95) death pathway. Following repeated cell cycles, $\mathrm{CD}^{+} \mathrm{T}$ cells regulate themselves through Fas-mediated apoptosis (34). Expression of Fas ligand (FasL) is induced following TCR occupancy, and is re- 
quired to engage Fas, and to trigger target cell death by apoptosis, a process known as activation-induced cell death (AICD; 35). Purified CD4 ${ }^{+}$T cells from $T$. cruzi-infected mice undergo AICD following TCR ligation (29), and upregulate Fas and FasL mRNA and their functional products (36). If these $\mathrm{CD} 4{ }^{+} \mathrm{T}$ cells are ligated with anti-TCR in the presence of $T$. cruzi-infected macrophages, a large increase in parasite replication and spread ensues (37). While ligation of Fas promotes lymphocyte apoptosis, increases parasite replication and eliminates most of the T-cell IFN- $\gamma$ production, blockade of FasL prevents T-cell apoptosis, and reduces parasite replication (37). In agreement with these findings, $\mathrm{CD}^{+} \mathrm{T}$ cells from FasLdeficient gld mice infected with $T$. cruzi are unable to die by AICD, and cannot exacerbate parasite growth in wild-type macrophages (37). These results describe a mechanism whereby the parasite takes advantage of T-cell apoptosis to replicate and spread in host tissues, in spite of a vigorous immune response against it. Recent data indicate that molecules isolated from $T$. cruzi can also directly induce host cell apoptosis under certain conditions. A purified ceramide-containing glycolipid isolated from $T$. cruzi (38) suppresses T-cell activation (39) and induces apoptosis in $\mathrm{CD}^{+} \mathrm{T}$ cells from infected, but not from control mice (Nascimento DO and DosReis GA, unpublished results). It also induces apoptosis in host macrophages in the presence of the cytokine IFN- $\gamma$, promoting the release of viable intracellular forms of the parasite (40). The ability of a T. cruzi molecule to trigger macrophage apoptosis and parasite release in the presence of IFN- $\gamma$, which is a powerful trypanocidal lymphokine, indicates another mechanism of escape from cell-mediated immunity. In fact, the remarkable ability of $T$. cruzi to persist indefinitely in the host despite a vigorous immune response shows that it has evolved mechanisms to cope with the effects of IFN$\gamma$. One widely recognized effector mech- anism against $T$. cruzi in infected mice is the production of nitric oxide (NO) by inducible NO synthase (NOS-2), a mechanism induced by synergism between IFN- $\gamma$ and TNF- $\alpha$. However, even though NO production has a protective role in infection by $T$. cruzi (41), recent studies also demonstrated parasiteprotective effects of NO. A recent review reported that $\mathrm{NO}$ increases intracellular replication of $T$. cruzi (42), and another study showed that IFN- $\gamma$-mediated NO release suppresses T-cells from infected mice (43). Moreover, NO production is involved in lymphocyte apoptosis during $T$. cruzi infection (44). These results show that, at least for T. cruzi, one major effector molecule in the type 1 response against the parasite (NO) is also involved in host-deleterious mechanisms such as T-cell unresponsiveness and death, and therefore, plays a "double-edged sword" role in host protection.

Recent studies demonstrated that phagocytosis of apoptotic cells deactivates macrophages through the engagement of scavenger cell surface receptors and production of anti-inflammatory mediators, such as prostaglandins and TGF- $\beta$ (45), further underscoring the general anti-inflammatory role of apoptosis in the immune system. Recent evidence indicates that phagocytosis of apoptotic T cells increases $T$. cruzi growth in macrophages in vitro and exacerbates parasitemia in vivo (Freire-de-Lima CG, DosReis GA and Lopes MF, unpublished results). One important remaining issue about apoptosis in Chagas' disease is whether it plays a role in the tissue lesions found in the heart tissue of the host (30).

Infection with the intracellular protozoan Toxoplasma gondii is another example of a situation in which regulation of host cell apoptosis plays a critical role. Infection with $T$. gondii induces apoptosis of host $\mathrm{CD}^{+} \mathrm{T}$ lymphocytes (46), which may involve a cooperative effect of IFN- $\gamma$ on Fas-mediated cell death (47). Virulent strains of $T$. gondii spread in the host by inducing host cell 
(macrophage) apoptosis (48). On the other hand, protective host $\gamma \delta$ T cells act by inducing hsp65 in infected macrophages, a protein that prevents macrophage apoptosis and spread of Toxoplasma infection (48). The opposite effect has also been reported. The intracellular parasite Leishmania donovani inhibits macrophage apoptosis through GMCSF production induced by its glycoconjugate lipophosphoglycan (LPG) (49). Intracellular parasites need to block host cell apoptosis to increase the likelihood of their survival, but in other situations they also need to induce apoptosis to spread infection. Probably, different parasite molecules are engaged differentially in these opposite responses. The complexity of host cell PCD induction also results from different possible consequences of the killing of an infected host cell. Whether the outcome of microbial release is beneficial or deleterious for the host depends on the biological properties of the pathogen, on its location, and on the inflammatory setting associated with the infection. Human monocytes infected with Mycobacterium tuberculosis are killed by distinct mechanisms by two different types of T-cell clones. While granule-mediated cytotoxicity by cytolytic T cells induces bacterial damage, Fas-mediated monocyte apoptosis induced by double-negative $\mathrm{T}$ cells results in the release of viable bacteria (50). It must be stressed that release of viable bacteria could facilitate their uptake by fresh mononuclear phagocytes better equipped for intracellular killing. In the case of motile parasites, however, release of viable organisms is associated with spread and virulent infection (48). Taken together, the data discussed in this section highlight the importance of studying host cell apoptosis in parasitic infections, and indicate that intervention in apoptosis could lead to important modifications of host-parasite interactions during chronic infections.

\section{References}

1. White $E$ (1996). Life, death and the pursuit of apoptosis. Genes and Development, 10: 1-15.

2. Thompson CB (1995). Apoptosis in the pathogenesis and treatment of diseases. Science, 267: 1456-1462.

3. Nagata S (1997). Apoptosis by death factor. Cell, 88: 355-365.

4. Shapiro J A (1995). The significance of bacterial colony patterns. BioEssays, 17: 597607.

5. Palkova Z, J anderova B, Gabriel J , Zkanova B, Pospisek M \& Forstova J (1997). Ammonia mediates communication between yeast colonies. Nature, 390: 532-536.

6. Barcinski MA \& Moreira MEC (1994). Cellular response of protozoan parasites to host-derived cytokines. Parasitology Today, 10: 352-355.

7. Moreira MEC, Del Portillo HA, Milder RV, Balanco J M \& Barcinski MA (1996). Heat shock induction of apoptosis in promastigotes of the unicellular organism Leishmania (L) amazonensis. J ournal of Cellu- lar Physiology, 167: 305-313.

8. Ameisen J C, Idziorek T, Billot-Mulot $\mathrm{O}$, Loyens $M$, Tissier J , Potentier A \& Ouassi A (1995). Apoptosis in a unicellular eukaryote (Trypanosoma cruzi): implications for the evolutionary origin and role of programmed cell death in the control of cell proliferation, differentiation and survival. Cell Death and Differentiation, 2: 285-300.

9. Welburn SC, Dale C, Ellis D, Beecroft R, Pearson TW \& Maudlin I (1996). Apoptosis in procyclic Trypanosoma brucei rhodesiense in vitro. Cell Death and Differentiation, 3: 229-235.

10. Murphy NB \& Welburn SC (1997). Programmed cell death in procyclic Trypanosoma brucei rhodesiense is associated with differential expression of mRNAs. Cell Death and Differentiation, 4: 365-370.

11. Kroemer G, Zamzani N \& Susin SA (1997). Mitochondrial control of apoptosis. Immunological Reviews, 18: 44-51.

12. Kroemer G (1997). Mitochondrial implication in apoptosis. Towards an endosymbiont hypothesis of apoptosis evolution.
Cell Death and Differentiation, 4: 443-456.

13. Kluck RM, Bossy-Wetzel E, Green DR \& Newmeyer DD (1997). The release of cytochrome $\mathrm{c}$ from mitochondria: a primary site for bcl-2 regulation of apoptosis. Science, 275: 1132-1136.

14. Barcinski MA, Schehtman D, Quintao LG, Costa DA, Soares LRB, Moreira MEC \& Charlab R (1992). Granulocyte-macrophage colony stimulating factor increases the infectivity of Leishmania amazonensis by protecting promastigotes from heat-induced death. Infection and Immunity, 60: 3523-3527.

15. Barcinski MA (1998). Apoptosis in Trypanosomatids: evolutionary and phylogenetic considerations. Genetics and Molecular Biology, 21: 21-24.

16. Vickerman K (1994). Evolutionary expansion of the trypanosomatid flagellates. International J ournal of Parasitology, 24: 1317-1331.

17. Maudlin I \& Welburn SW (1994). Maturation of Trypanosome infections in tsetse. Experimental Parasitology, 79: 202-205. 
18. Welburn SC, Barcinski MA \& Williams GT (1997). Programmed cell death in Trypanosomatids. Parasitology Today, 13: 2226.

19. Tibayrenc M (1995). Population genetics of parasitic protozoa and other microorganisms. Advances in Parasitology, 36: 47-115.

20. Fernandes AP, Nelson $K \&$ Beverley SM (1993). Evolution of nuclear ribosomal RNAs in kinetoplastid Protozoa: Perspectives on the age and origins of parasitism. Proceedings of the National Academy of Sciences, USA, 90: 11608-11612.

21. Maudlin I (1997). Life and death in Trypanosoma brucei rhodesiensis. Memórias do Instituto Oswaldo Cruz, 92 (Suppl I): 7 (Abstract).

22. Fearon DT \& Locksley RM (1996). The instructive role of innate immunity in the acquired immune response. Science, 272 : 50-54.

23. J ulia V, Rassoulzadegan $M \&$ Glaichenhaus N (1996). Resistance to Leishmania major induced by tolerance to a single antigen. Science, 24: 421-423.

24. Picot S, Burnod J, Bracchi V, Chumpitazi BFF \& Ambroise-Thomas P (1997). Apoptosis related to chloroquine sensitivity of the human malaria parasite Plasmodium falciparum. Transactions of the Royal Society of Tropical Medicine and Hygiene, 91: 590-591.

25. Cuff S \& Ruby J (1996). Evasion of apoptosis by DNA viruses. Immunology and Cell Biology, 74: 527-537.

26. Fugier-Vivier I, Server-Delprat C, Rivailler $P$, Rissoan MC, Liu YJ \& RabourdinCombe C (1997). Measles virus suppresses cell-mediated immunity by interfering with the survival and functions of dendritic and $T$ cells. J ournal of Experimental Medicine, 186: 813-823.

27. Zychlinsky A \& Sansonetti P (1997). Apoptosis in bacterial pathogenesis. J ournal of Clinical Investigation, 100: 493-495.

28. Zhang J , Andrade ZA, Andrade S, Takeda K, Sadigursky M \& Ferrans VJ (1997). Apoptosis in a canine model of acute chagasic myocarditis. Memórias do Instituto Oswaldo Cruz, 92 (Suppl I): 107 (Abstract).

29. Lopes MF, Veiga VF, Santos AR, Fonseca MEF \& DosReis GA (1995). Activationinduced $C D 4^{+} \mathrm{T}$ cell death by apoptosis in experimental Chagas' disease. J oumal of Immunology, 154: 744-752.

30. DosReis GA, Fonseca MEF \& Lopes MF (1995). Programmed T-cell death in experimental Chagas disease. Parasitology Today, 11: 390-394.
31. Lenardo MJ (1991). Interleukin-2 programs mouse $\alpha ß$ T lymphocytes for apoptosis. Nature, 353: 858-861.

32. Fucs $R \&$ Barcinski MA (1987). Effect of hydroxyurea on the concanavalin A proliferative response of Trypanosoma cruziinfected mice. Immunology Letters, 15: 317-321.

33. Lopes MF \& DosReis GA (1996). Trypanosoma cruzi-induced immunosuppression: selective triggering of $\mathrm{CD}^{+}{ }^{+} \mathrm{T}$ cell death by the T-cell receptor-CD3 pathway and not by the CD69 or Ly-6 activation pathway. Infection and Immunity, 64: 15591564.

34. Alderson MR, Tough TW, Davis-Smith T, Braddy S, Falk B, Schooley KA, Goodwin RG, Smith CA, Ramsdell F \& Lynch DH (1995). Fas ligand mediates activation-induced cell death in human T lymphocytes. J ournal of Experimental Medicine, 181: 71-77.

35. Van Parijs L \& Abbas AK (1996). Role of Fas-mediated cell death in the regulation of immune responses. Current Opinion in Immunology, 8: 355-361.

36. Lopes MF, Nunes MP, Henriques-Pons A, Giese NA, Morse III HC, Davidson W, Araújo-J orge TC \& DosReis GA (1998). Increased susceptibility of Fas ligand-deficient gld mice to Trypanosoma cruzi infection due to a Th2-biased host immune response. European J ournal of Immunology, 28 (in press).

37. Nunes MP, Andrade RM, Lopes MF \& DosReis GA (1998). Activation-induced T cell death exacerbates Trypanosoma cruzi replication in macrophages cocultured with $\mathrm{CD}^{+} \mathrm{T}$ lymphocytes from infected hosts. J ournal of Immunology, 160: 13131319.

38. Previato J O, Gorin PAJ, Mazurek M, Xavier MT, Fournet B, Wieruszesk J M \& Mendonça-Previato L (1990). Primary structure of the oligosaccharide chain of lipopeptide phosphoglycan of epimastigote forms of Trypanosoma cruzi. J ournal of Biological Chemistry, 265: 2518-2526.

39. Gomes NA, Previato J O, Zingales B, Mendonça-Previato $L$ \& DosReis GA (1996). Downregulation of T-lymphocyte activation in vitro and in vivo induced by glycoinositol phospholipids from Trypanosoma cruzi: assignment of the T-cell suppressive determinant to the ceramide domain. J ournal of Immunology, 156: 628635.

40. Freire-de-Lima CG, Nunes MP, Corte-Real S, Soares MP, Previato J O, MendonçaPreviato L \& DosReis GA (1998). Pro- apoptotic activity of a Trypanosoma cruzi ceramide-containing glycolipid turned on in host macrophages by IFN- $\gamma$. J ournal of Immunology, 161: 4909-4916.

41. Vespa GN, Cunha FQ \& Silva J S (1994). Nitric oxide is involved in control of Trypanosoma cruzi-induced parasitemia and directly kills the parasite in vitro. Infection and Immunity, 62: 5177-5182.

42. Rottenberg ME \& Öm A (1997). Conflicting roles of NO in Trypanosoma cruzi infection. Immunologist, 5: 127-132.

43. Abrahamsohn IA \& Coffman RL (1995). Cytokine and nitric oxide regulation of the immunosuppression in Trypanosoma cruzi infection. J ournal of Immunology, 155: 3955-3963.

44. Martins GA, Cardoso MA, Aliberti JC \& Silva J $S$ (1998). Nitric oxide-induced apoptotic cell death in the acute phase of Trypanosoma cruzi infection in mice. Immunology Letters, 63: 113-120.

45. Fadok VA, Bratton DL, Konowal A, Freed PW, Westcott J Y \& Henson PM (1998). Macrophages that have ingested apoptotic cells in vitro inhibit proinflammatory cytokine production through autocrine/paracrine mechanisms involving TGF-ß3, PGE-2 and PAF. J ournal of Clinical Investigation, 101: 890-898.

46. Khan IA, Matsuura T \& Kasper LH (1996). Activation-mediated $\mathrm{CD}^{+}{ }^{+} \mathrm{T}$ cell unresponsiveness during acute Toxoplasma gondii infection in mice. International Immunology, 8: 887-896.

47. Liesenfeld O, KosekJ C \& Suzuki Y (1997). Gamma interferon induces Fas-dependent apoptosis of Peyer's patch T cells in mice following peroral infection with Toxoplasma gondii. Infection and Immunity, 65: 4682-4689.

48. Hisaeda $\mathrm{H}$, Sakai $\mathrm{T}$, Ishikawa $\mathrm{H}, \mathrm{Maekawa}$ $Y$, Yasutomo K, Good RA \& Himeno K (1997). Heat shock protein 65 induced by gamma-delta $T$ cells prevents apoptosis of macrophages and contributes to host defense in mice infected with Toxoplasma gondii. J ournal of Immunology, 159: 2375-2381.

49. Moore KJ \& Matlashewski G (1994). Intracellular infection by Leishmania donovani inhibits macrophage apoptosis. J ournal of Immunology, 152: 2930-2937.

50. Stenger S, Mazzaccaro RJ, Uyemura K, Cho S, Barnes PF, Rosat JP, Sette A, Brenner MB, Porcelli SA, Bloom BR \& Modlin RL (1997). Differential effects of cytolytic T cell subsets on intracellular infection. Science, 276: 1684-1687. 